\title{
Bern und die Westschweiz: eigentlich so nah ...
}

\section{Monique Gauthey}

Fachärztin, ehemaliges Mitglied des Zentralvorstands der FMH

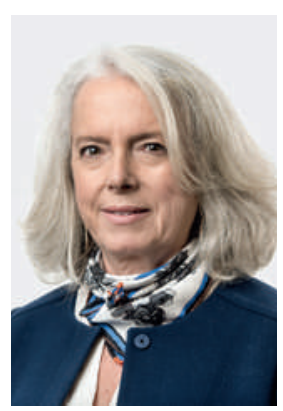

In den acht Jahren als Pendlerin auf der Strecke GenfBern erschien mir die Fahrt im Laufe der Zeit immer lohnender, vor allem aber erschloss sich mir die Bedeutung der täglichen Aktivitäten der FMH stärker. Dennoch zieht es nur wenige Genferinnen und Genfer in die Hauptstadt. Schweizerdeutsch wird zwar bei Arbeitssitzungen so gut wie nie gesprochen, hindert jedoch in der Praxis viele Westschweizer am Austausch mit unseren alemannischen Freunden. Dieses Handicap dürfte so manchen entmutigen ... Von Genf aus erscheint Europa viel näher als die Bundeshauptstadt, und die Wellen der französischen Politik schwappen bis an unsere Türen. In Bern wurden mir hingegen die verschlungenen Pfade der Schweizer Bundespolitik, ihre impliziten Regeln und Machtgeflechte sowie die Gewichtung der verschiedenen Regionen bewusst.

\section{Von Genf aus betrachtet scheint Europa viel näher als die Bundeshauptstadt.}

In der Schweiz arbeitet jeder fünfte Arzt in einem frankophonen Kanton und jeder elfte in Genf. Die Orientierung des Genfers in Richtung Frankreich, die Verbindungen mit der anglophonen Welt und den internationalen Organisationen unterstreichen die Besonderheiten der Genfer Medizin noch zusätzlich.

Allerdings sollten die in Bern erarbeiteten politischen Lösungen für Ärztinnen und Ärzte die Besonderheiten jeder Region berücksichtigen. Ihre Arbeit vor Ort ist immer stark geprägt vom regionalen Gesundheitsnetz eines Kantons und davon, wie sich dieses im Laufe der Geschichte entwickelt hat. Die FMH ist ein pluralistischer Verband, wie es europaweit nur wenige gibt. Sie ist Standesorganisation, Gewerkschaft und politisch-akademische Denkfabrik zugleich. Die FMH ist in vielen ethischen und deontologischen Bereichen aktiv, aber auch in den Bereichen Prävention, Leistungsqualität, Statistik und Ärztedemographie. Sie kümmert sich auch um Tarifsysteme zur Abgeltung ärztli- cher Leistungen und engagiert sich für günstige rechtliche Rahmenbedingungen im ärztlichen Berufsalltag. Zentralvorstand, Delegiertenversammlung oder Ärztekammer: Alle Kräfte sind gefragt, wenn es darum geht, die anstehenden Aufgaben zu stemmen.

Die Reduzierung der Zentralvorstandsmitglieder von neun auf sieben - dies hat die Ärztekammer im Oktober 2015 beschlossen - hat unmittelbar zur Folge, dass die Westschweizer und Tessiner Mitglieder schlechter repräsentiert sind. Der Beschluss spiegelt aber auch eine allgemeine Entwicklung wider, nämlich die vom «Bürger im Einsatz für das Allgemeinwohl» hin zum «vermehrt konsumorientierten Mitglied» - eine Negativtendenz, die sich in der gesamten Zivilgesellschaft feststellen lässt. Durch die gleichzeitige Reduzierung der Mitglieder des Zentralvorstands und der Anzahl der Sitzungen der Delegiertenversammlung sollen Einsparungen erzielt werden, um dringende politische Aktionen zu finanzieren. Damit bewegen wir uns von der aktiven Einbeziehung unserer Mitglieder hin zu einer passiven Politik, die sich an eventuellen positiven Auswirkungen einer Medienkampagne orientiert.

Die politischen Lösungen müssen die medizinischen Besonderheiten jeder einzelnen Region berücksichtigen.

Durch diesen subtilen Schwenk geraten die Westschweizer Ärzte ins Hintertreffen, und zwar zu einem Zeitpunkt, wo die Verkomplizierung des Gesundheitswesens gerade mehr Einbeziehung auf allen Ebenen, in allen Kommissionen und Arbeitsgruppen rund um die FMH erfordern würde. Durch eine zu schwache Westschweizer Vertretung in unseren Führungsorganen kommt uns auch ein wertvolles Netzwerk an Ärzten mit ihren jeweiligen Kontakten abhanden.

Nun, da ich mich zurückziehe, kann ich nur dafür plädieren, dass die Westschweizer Ärzte sich mobilisieren, auch wenn dazu die Türen der FMH etwas aufgestossen werden müssen. 